Materials at Elevated Temperatures by an Energy Approach Using Direct Observation and Compliance Calculation of the Crack Length," J. Mater. Sci., 20, 2586-96 (1985).

'R. F. Cook, B. R. Lawn, and C. J. Fairbanks "Microstructure-Strength Properties in Ceramics: I, Effect of Crack Size on Toughness," J. Am. Ceram. Soc., 6 [11] 604-15 (1985).

${ }^{6}$ S. M. Wiederhorn, B. J. Hockey, R. F. Krause, Jr., and K. Jakus, "Creep and Fracture of Vitreous-Bonded Aluminum Oxide," J. Mater. Sci., 21, 810-24 (1986).

D. Broek, Elementary Engineering Fracture Mechanics; pp. 115-16, 185-91 Noordhoff International Publishing, Leyden, The Netherlands, 1974.
${ }^{8} \mathrm{D}$. Broek, "The Energy Criterion for Fracture of Sheets Containing Cracks," Appl. Mater. Res.. 4, 188-89 (1965).

${ }^{9}$ D. B. Marshall and B. R. Lawn, "Residual Stress Effects in Sharp Contact Cracking: Part 1, Indentation Fracture Mechanics,"J. Mater. Sci., 14, 2001-12 (1979).

${ }^{10} \mathrm{~J}$. Mandel, The Statistical Analysis of Experimental Data. Dover Publications, New York, 1984.

${ }^{11}$ B. R. Lawn and D. B. Marshall, "Hardness, Toughness, and Brittleness: An Indentation Analysis," J. Am. Ceram. Soc., 62 [7] 347-50 (1979).

${ }^{2}$ J. C. Newman, Jr., and I. S. Raju, "An Empirical Stress-Intensity Factor Equation for the Surface Crack," Eng. Fract. Mech., 15, 185-92 (1981).

\title{
Transformation Plasticity of $\mathrm{CeO}_{2}$-Stabilized Tetragonal Zirconia Polycrystals: I, Stress Assistance and Autocatalysis
}

\author{
PATRICIO E. REYES-MOREL ${ }^{\star,+,+, 8}$ and I-WEI CHEN ${ }^{\star, \dagger}$ \\ Department of Materials Science and Engineering and Department of Nuclear Engineering, University of Michigan, \\ Ann Arbor, Michigan 48109-2136
}

Transformation plasticity in $\mathrm{CeO}_{2}$-stabilized tetragonal zirconia polycrystals due to the tetragonal-to-monoclinic transformation was studied by inducing volumetric and shear deformation under compression and bending between the burst temperature of martensite (monoclinic) formation $\left(\boldsymbol{M}_{b}\right)$ and the burst temperature of austenite (tetragonal) formation $\left(A_{b}\right)$. The stress-strain curve features a load drop, a perfect plastic regime, and an extended strain-hardening regime before the exhaustion of transformation. Macroscopic shear bands formed in the perfect plastic regime. The yield stress has a strong, positive pressure and temperature sensitivity but is strain-rate sensitive only in the last stage of deformation. These results are rationalized in terms of stress assistance to the transformation which, in a homogeneous tetragonal polycrystal, may propagate autocatalytically. Autocatalysis can be impeded by a second phase, such as monoclinic $\mathrm{ZrO}_{2}$ or $\mathrm{Al}_{2} \mathrm{O}_{3}$, and is suppressed at higher temperature. Flow localization is found to precede and precipitate crack formation. As a result, the actual fracture energy is much less than the total plastic work. The implications of stress-assisted, autocatalytic transformation on strength and toughness are explored.

\section{Introduction}

T RANSFORMATION toughening in zirconia-containing ceramics is due to a phase transformation which occurs near the crack tip.' Mechanically, a transformation-induced plastic deformation, i.e., transformation plasticity, provides the requisite energy dissipation and stress-field shielding for toughening. Detailed knowledge of transformation plasticity, especially of its constitutive behavior, is essential for an understanding of transformation toughening. The present paper reports our study of transformation plasticity in $\mathrm{CeO}_{2}$-stabilized tetragonal zirconia polycrystals (Ce-TZPs), which have a microstructure of homogeneous tetragonal $(t)$ grains. ${ }^{2}$ This type of microstructure is commonly found in

Manuscript No. 199776. Received August 27, 1987; approved November 23, 1987 Presented at the 88th Annual Meeting of the American Ceramic Society, Chicago IL, April 28, 1986 (Basic Science Division, Paper No. 39-B-86).

Supported by the National Science Foundation under Grant No, DMR $8407868 / 8609146$. Partial support for P. E. Reyes-Morel was provided by the Chilean Government.

${ }^{*}$ Member, the American Ceramic Society.

Department of Materials Science and Engineering.

Department of Nuclear Engineering.

${ }^{\$}$ Now with the Chilean Nuclear Energy Commission, Santiago, Chile. fine-grain, partially stabilized zirconia ceramics.

To establish the connection between transformation, transformation plasticity, and transformation toughening, two aspects of transformation plasticity are deemed critical. ${ }^{3}$ First, the stress assistance to transformation must be demonstrated; this incorporates the mechanical driving force along with the chemical driving force in the analysis of stress-assisted transformation. Second, the stress-state effect on transformation must be investigated to formulate a constitutive relation to be used for the crack-tip analysis. As will be demonstrated in the present study, the temperature and stress-state dependence of transformation plasticity can provide the aforementioned information. In simplest terms, the stress-strain curve has a one-to-one correspondence to the temperaturetransformation curve, with the magnitude of the flow stress being determined by the stress state and the deformation temperature.

A typical transformation curve of Ce-TZP is shown in Fig. 1, which plots linear dilatation versus the temperature. In the present material, the tetragonal-to-monoclinic $(m)$ transformation, when

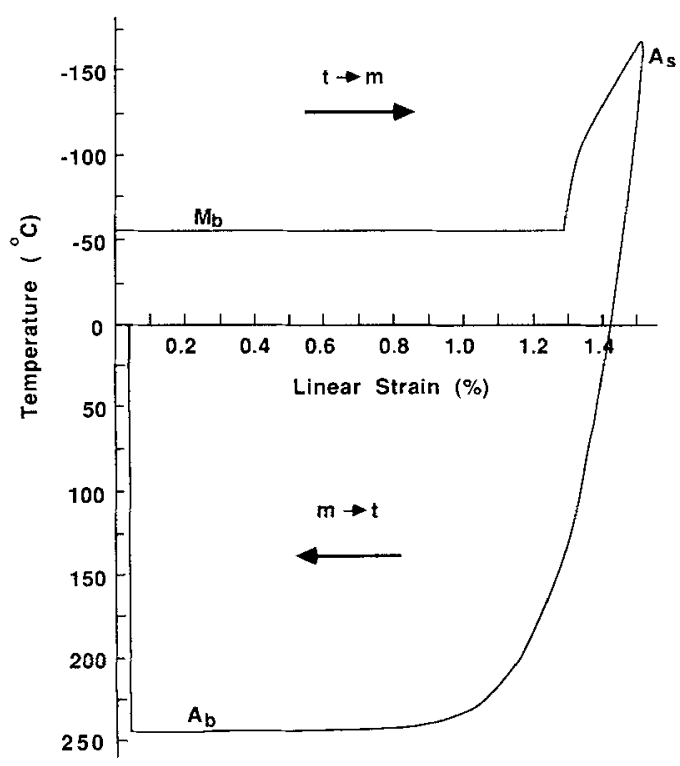

Fig. 1. Dilatometry curve of Ce-TZP (grain size $=$ $1.61 \mu \mathrm{m})$ 

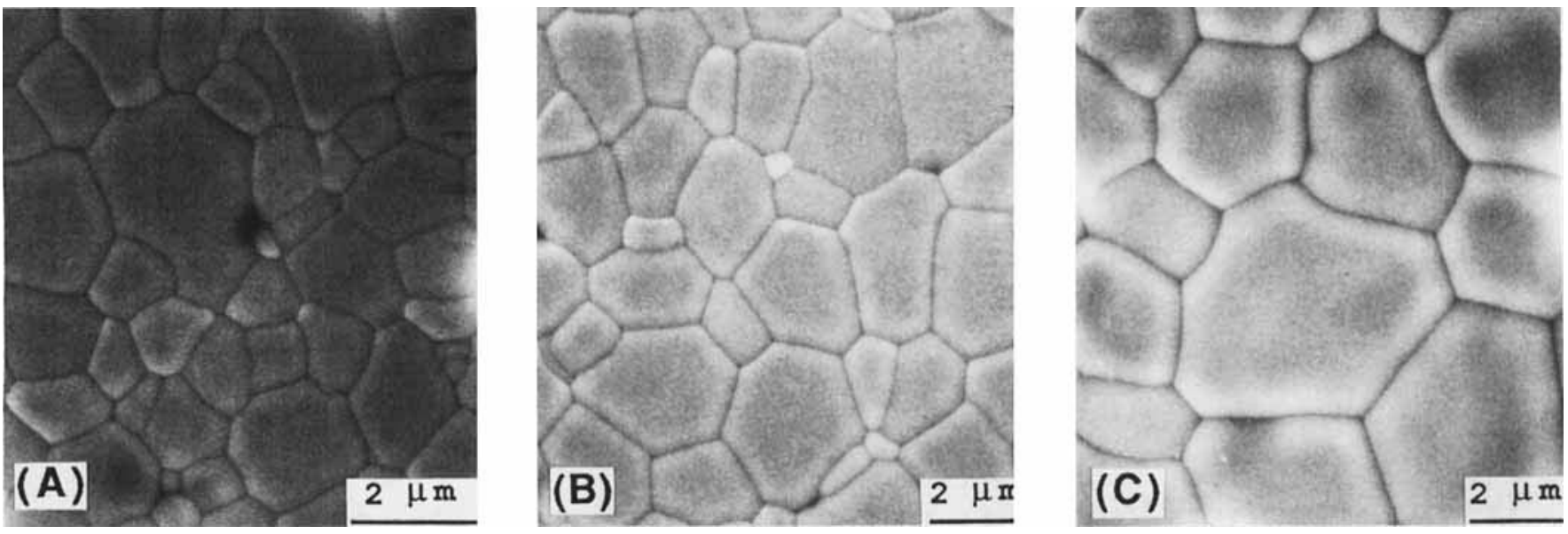

Fig. 2. Scanning electron micrographs of Ce-TZP with grain size of $(A) 0.95,(B) 1.61$, and $(C) 2.80 \mu \mathrm{m}$.

completed, causes a 5 vol\% dilatation, or $1.67 \%$ linear expansion. The metastable tetragonal polycrystal can be cooled to the burst temperature of martensite (monoclinic) formation $\left(M_{h}\right)$ when a sudden burst of $t$-to- $m$ transformation takes place. Linear expansion of $\approx 1 \%$ results from the burst. Further cooling caused additional transformation, sometimes with smaller bursts, at lower temperatures. Upon heating, the reverse transformation begins slowly at a rather low start temperature of austenite (tetragonal) formation $\left(A_{s}\right), A_{s}<M_{b}$. The reverse transformation gradually accelerates as heating continues until an $m$-to- $t$ burst occurs at the burst temperature of austenite $\left(A_{b}\right)$. Typically, the $m$-to- $t$ burst is smaller than the $t$-to- $m$ burst. However, the total $m$-to- $t$ reversion from $A_{s}$ to $A_{b}$ is $>90 \%$ of the total $t$-to- $m$ transformation. Further heating results in little change. The above transformation is driven by the chemical force only. If, in addition, an external stress is applied to provide part of the driving force, then a stress increase may have the same effect as a temperature decrease. Assuming such a correspondence, we envision the stress-strain curve to have a profile similar to that of the temperature-strain curve as shown in Fig. 1. This correspondence, under various stress states, will be explored between $M_{b}$ and $A_{b}$ in the present study. Transformation plasticity which satisfies the above correspondence will be termed stress assisted.

One important feature apparent in Fig. 1 is the presence of two distinct stages of transformation, namely the burst at $M_{b}$ and the subsequent gradual transformation below $M_{b}$. It will be shown in this study that the burst corresponds to a perfect plastic stage in transformation plasticity, and the subsequent transformation is manifested as a strain-hardening stage. The presence of the burst is a unique characteristic of metastable tetragonal polycrystals typified by the Ce-TZP studied here, in which martensitic transformation may propagate over an extended region without interruption. Indeed, the burstlike transformation will be termed autocatalytic; its origin is probed in the present study. The burst is terminated when a substantial portion of the polycrystal has transformed, leaving the $t$ phase partitioned into isolated metastable domains surrounded by a stable monoclinic matrix. The latter microstructure rather resembles a two-phase, dispersed zirconia composite, such as magnesium-partially-stabilized zirconia (Mg-PSZ) or alumina containing tetragonal zirconia (ZTA), in which transformation, as evidenced by both the temperaturetransformation curve ${ }^{4}$ and the stress-strain curve ${ }^{3}$ is gradual and without a burst. Since both microstructures occur in Ce-TZP, we expect its transformation and deformation to evolve in two stages accordingly.

The deformation above $A_{b}$ has very different characteristics. It also involves the recovery of shape strain and microstructure similar to those obtained after annealing. This behavior of reverse transformation, along with the nature of strain accommodation in transformation plasticity, and the grain-size dependence of trans- formation temperatures and stresses, will be the subject of a companion paper.

\section{Experimental Procedure}

The materials studied here are several Ce-TZP batches containing $12 \mathrm{~mol} \% \mathrm{CeO}_{2}{ }^{2}$ One batch with a grain size from 0.96 to $2.8 \mu \mathrm{m}$ was sintered between $1400^{\circ}$ and $1600^{\circ} \mathrm{C}$ using a commercial powder; the other batch with a grain size of $1.2 \mu \mathrm{m}$ and sintered at $1500^{\circ} \mathrm{C}$ was received in the as-sintered form from a different source. The compositions of the two batches are slightly different. The micrographs shown in Fig. 2 reveal a fine-grain, uniform microstructure with low porosity. The grains are made of $t$ phase only.

To establish a reference data base for the $t$-to- $m$ transformation, dilatometry curves of the type shown in Fig. 1 were obtained for all the studied materials. The two batches of materials were found to behave slightly differently in their transformation temperatures. In addition, as will be reported in the companion paper, a systematic grain-size dependence of the transformation temperatures was found within the same batch of materials. Despite the differences in transformation temperatures, we found the general mechanical and transformation characteristics of all materials to be similar. Indeed, their mechanical behavior can be normalized with respect to a reference taken to be $M_{b}$. In view of the similarity, the samples reported below will be identified by their transformation temperatures only. Some results of alumina-containing Ce-TZP are also reported here to provide further insight into the role of microstructure.

Mechanical testing in this study was conducted utilizing several techniques. In addition to standard compression tests, modified compression tests were performed in the surrounding of an independently controlled pressure (hydraulic-compression tests). ${ }^{6}$ The latter technique is uniquely suitable for the study of stress-state (pressure) sensitivity of transformation plasticity. Specifically, it provides, independently, an adjustable pressure component in the stress state. ${ }^{3}$ Another advantage in using this technique for testing ceramics is the pressure effect on stabilization of microcracks, so that premature failure can be avoided. ${ }^{3}$ In the present study, a full range of transformation strains to $4.5 \%$ has been obtained. Further details of the hydraulic-compression test were documented elsewhere. $^{7}$ In addition to compression tests, the tensile surface in four-point bending was studied.

Transformation strains, in shear and dilatation, were recorded by axial and radial strain gages. As will become evident later, shear localization is prevalent in Ce-TZP at lower temperatures. In such cases, accurate strain measurements require relatively large specimens with oversized strain gages. This practice was followed whenever accurate stress-strain curves were sought. The occurrence of macroscopic shear localization in the present material, how- 


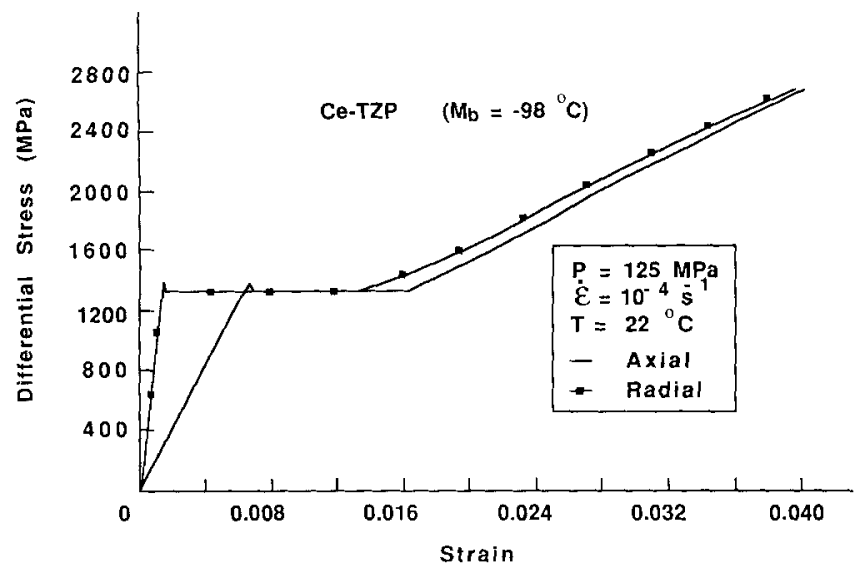

Fig. 3. Stress-strain curve in hydraulic compression at $125 \mathrm{MPa}$.

ever, is preceded by a sharp load drop. Thus, yield stress can always be measured accurately, irrespective of sample and gage sizes.

Standard optical and scanning electron microscopy and X-ray diffractometry using $\mathrm{Cu} K \alpha$ radiation were employed for microstructural observation and phase identification. Lattice constants of $t$ and $m$ phases were measured by $\mathrm{X}$-ray diffractometry using undeformed and nearly fully deformed/transformed specimens. The constraint on the monoclinic lattice in the latter case is small and neglected. ${ }^{8}$

\section{Results}

\section{(I) Stress-Strain Curves}

A typical stress-strain curve of Ce-TZP confined at $125 \mathrm{MPa}$ is shown in Fig. 3. Here, the vertical axis records the uniaxial compression stress, onto which a hydrostatic pressure is superimposed as indicated. This axial stress will be referred to as differential stress, denoted as $\Sigma$. The axial strain in the plot is compressive, whereas the radial strain is tensile. The plastic portion has two distinct stages: The first is a perfect plastic regime with a constant flow stress, which is preceded by a load drop at the end of the elastic range; the second is a strain-hardening regime with an increasing flow stress, which is terminated by fracture. The volumetric plastic strain is always positive and approaches $4.5 \%$ near fracture. Comparing the latter with the total lattice expansion during $t$-to- $m$ transformation, i.e., $5 \%$, we readily conclude that the tetragonal phase is nearly exhausted at fracture.

The above stress-strain curve is in qualitative correspondence with the temperature-strain curve shown in Fig. 1. The elastic range corresponds to the cooling portion during which the only strain is thermal. The yield point corresponds to $M_{b}$, at the onset of an abrupt burst of $t$-to- $m$ transformation. The subsequent strainhardening stage corresponds to the sub- $M_{b}$ transformation. Of course, the load drop is a special feature of mechanical testing in the perfect plastic regime. It can be attributed to the clastic unloading in response to rapid plastic straining at a constant stress. In Ce-TZP at room temperature or below, these events occurred so rapidly that a loud noise was always generated. It should be noted that, for the Ce-TZP studied here, even cooling to $4.2 \mathrm{~K}$ caused only a limited amount of transformation beyond the burst. In contrast, mechanically induced transformation proceeds much further, approaching the fully transformed state.

During four-point bending, the load-displacement curves are not linear at higher stresses. This has been previously noted by Tsukuma and Shimada. ${ }^{2}$ We also found that the local tensile strain on the tensile surface as recorded by the strain gage is not linear with the total loading point displacement, often experiencing sudden drops in coincidence with load drops as shown in Fig. 4. This indicates that deformation on the tensile surface is localized in nature, such that elastic unloading may cause contraction in regions outside the deformation bands. Serrated flow is thus evident

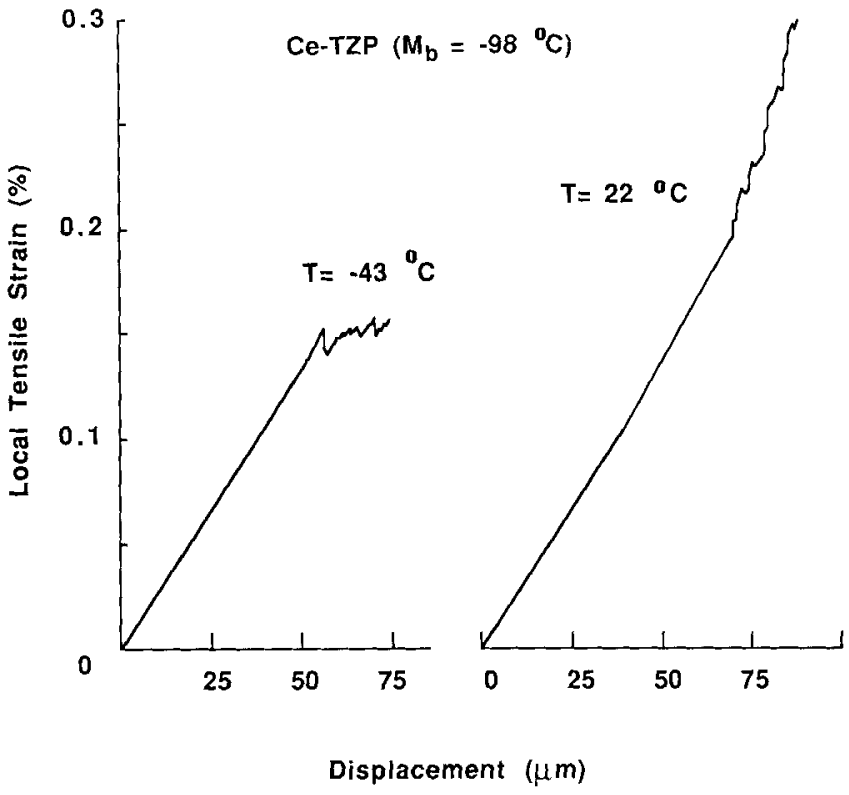

Fig. 4. Local tensile strain, recorded by a strain gage, versus displacement in four-point bending.

in all of the bending curves, becoming more pronounced as $M_{b}$ is approached. At higher temperatures, plastic flow was more stable. The failure strains, however, were rather small compared to those shown in Fig. 3. It should be noted that, because of the stress-state sensitivity and the limited ductility of transformation plasticity, only the tensile side of the bent specimen had yielded. Thus, no perfect plastic regime in the load-displacement curve is expected for bending. This asymmetry in yielding is also reflected, under the more-stable deformation condition at $22^{\circ} \mathrm{C}$ in Fig. 4 , in the strain-bend-displacement curve of which the postyielding portion is concave upward. This is consistent with a gradual shift of the neutral axis toward the less-deformable compressive side, resulting in more tensile strain and less compressive strain at a given bend displacement.

The compression stress-strain curve obtained at higher temperatures, such as the one shown in Fig. 5, has gradual yielding instead. A steady-state strain hardening seems to dominate over the

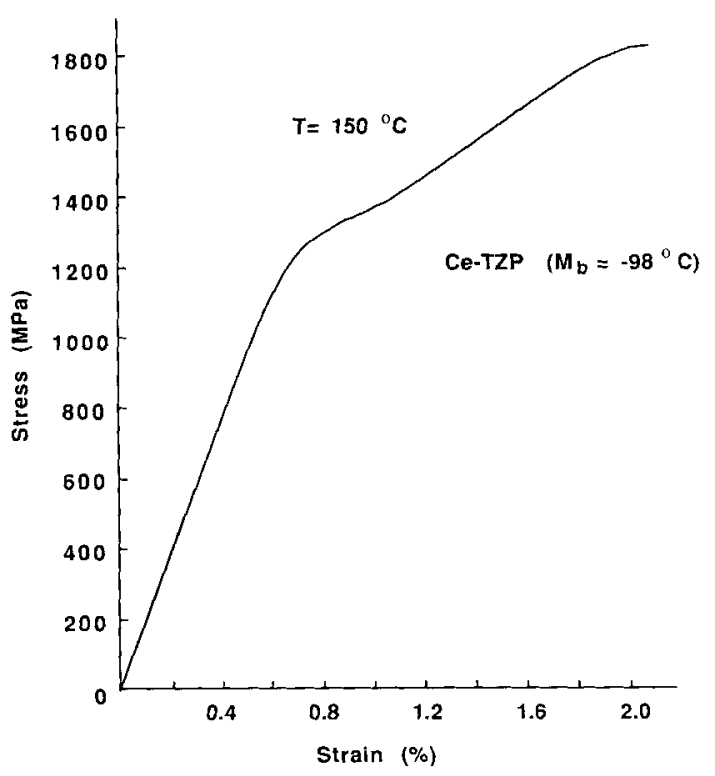

Fig. 5. Uniaxial compression stress--strain curve at $150^{\circ} \mathrm{C}$ for Ce-TZP. 


\section{$0.5 \mathrm{~mm}$}

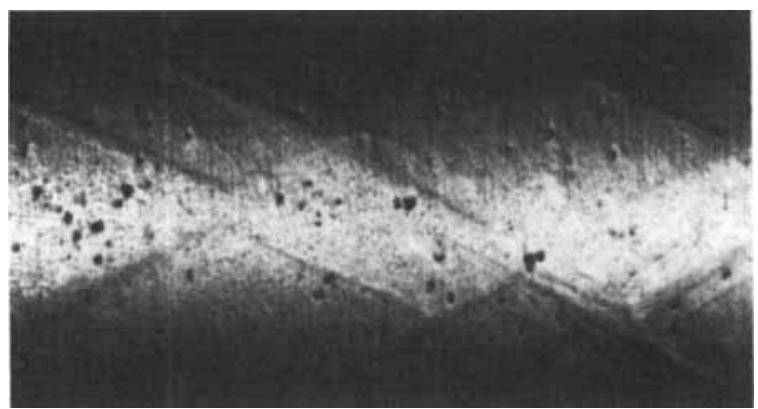

Fig. 6. Surface light micrograph of Ce-TZP deformed under hydraulic compression showing Luders bands. The angle between the band and the $z$ axis is $33^{\circ}$.

midstrain range. At still higher temperatures above $A_{b}$, although the stress-strain curve in loading is similar, the plastic strain recovers during unloading. This remarkable effect will be reported in the companion paper.
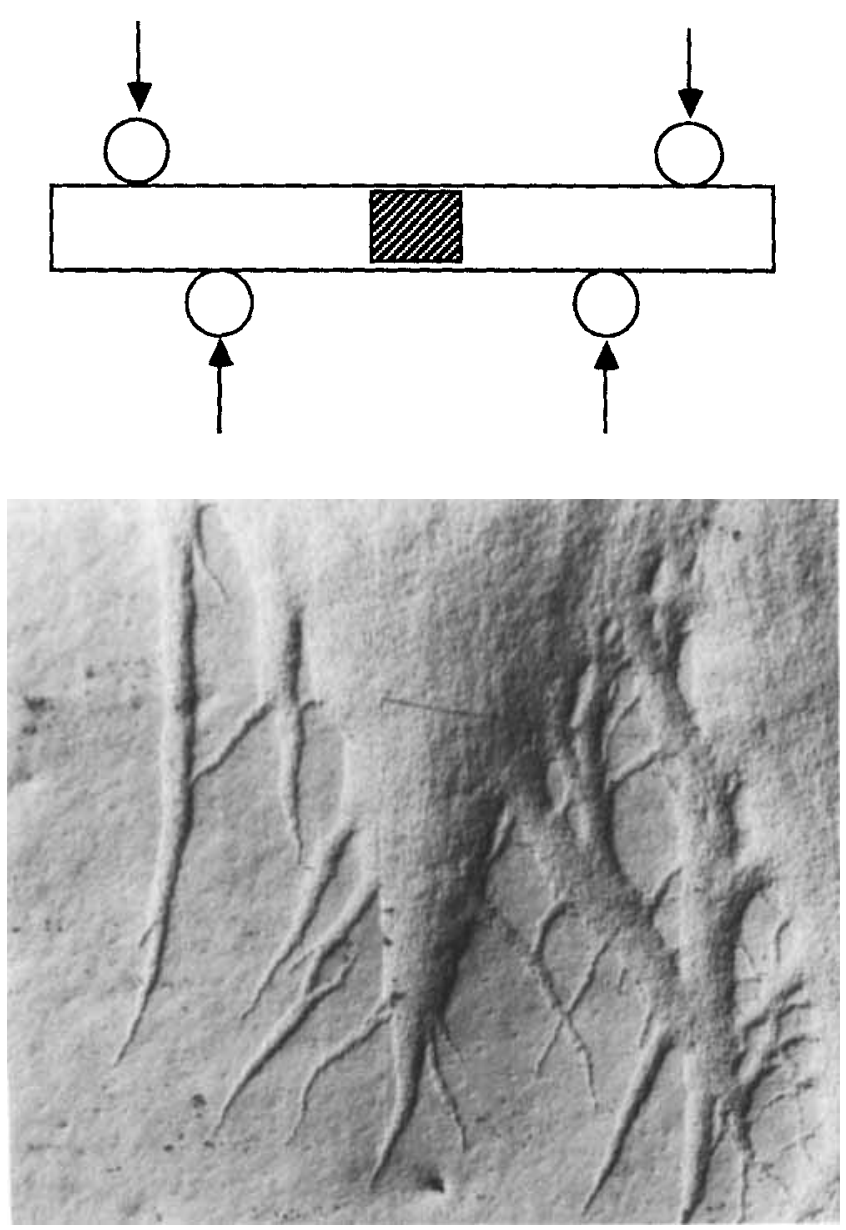

\section{$\mathrm{T}=202 \mathrm{~K}$}

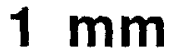

Fig. 7. Light micrograph of the side surface on the bend specimen.

\section{(2) Phase Identification, Texture, and Deformation Bands}

Standard X-ray diffraction using $\operatorname{Cu} K \alpha$ radiation was performed. Before deformation, Ce-TZP is entirely tetragonal. During compression, the amount of monoclinic phase increases as deformation proceeds. Comparison of the longitudinal and transverse sections revealed marked differences in diffracted intensities from various reflections between the two, indicating a texture of both the $m$ phase and the retained $t$ phase. An example of diffraction patterns of the two sections will be shown in the companion paper. ${ }^{5}$ For the bend specimens, monoclinic reflections were found on the tensile surface. The diffraction patterns of both phases again were different from those of the random powders and of the compression samples, suggesting yet another texture. On the compression side, only a tetragonal diffraction pattern, identical to the one of random powders, was observed. As reported elsewhere, the texture in the present case is primarily due to a coupling between applied stress and transformation shear strain. ${ }^{9}$ Since the tensile stress and the compressive stress on two sides of the bend specimen were of the same magnitude, the absence of transformation on the compression side provided direct evidence of the stress-state sensitivity of transformation plasticity favoring tension.

Surfaces of the hydraulically deformed samples were populated by a large number of deformation bands. They form a diamondshaped grid which is pointed and elongated along the stress axis, as shown in Fig. 6. Less-deformed samples had fewer deformation bands. The formation of deformation bands is another indication of deformation instability, which was already evidenced by the load drops and the flat, perfect plastic regime in the stress-strain curve. The orientation of these deformation bands suggests that they are dilatant-shear bands, ${ }^{10}$ as expected from the stress-strain behavior of Fig. 3.

On the bend specimens, deformation bands were detected on tensile, but not on compressive, surfaces. Figure 7, a micrograph of the side surface, provides a vivid illustration of this effect. The rootlike deformation bands emanate from the top, tensile side and propagate into the center span but do not penetrate through to the compressive side. (This asymmetry contrasts to the symmetric hingelike deformation bands under both tensile and compressive surfaces subject to normal bending in slip-controlled plasticity. ${ }^{12}$ The tips of the "roots" appeared to bifurcate, forming an obtuse angle with respect to the prevailing tensile-stress axis. Further analysis of deformation bands in transformation plasticity is postponed to a future paper.

\section{(3) Stress-State Dependence}

The uniaxial compressive-yield stress in hydraulic compression, i.e., $Y_{c}$, increases with confining pressure, $P$, as shown in Fig. 8. The data plotted here were for the first stage of plastic deformation, but a similar elevation of the flow stress was also found in the 


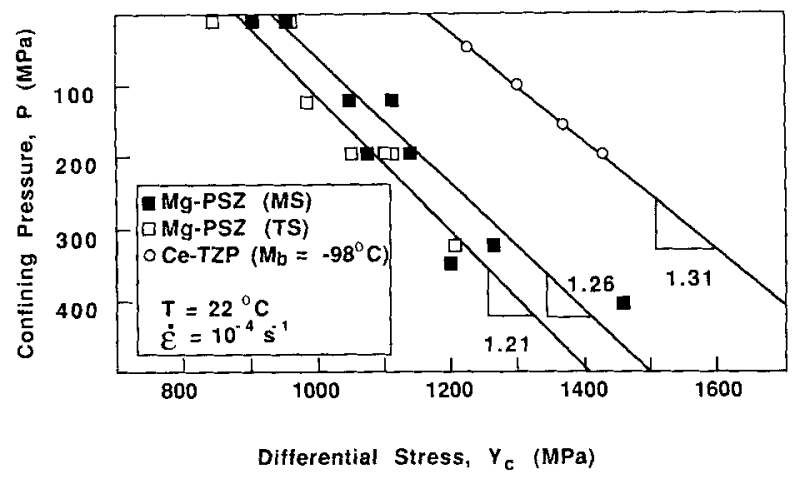

Fig. 8. Yield stress versus confining pressure for Mg-PSZ (MS), Mg-PSZ (TS), and Ce-TZP. The slopes of the lines are the pressure sensitivity of transformation plasticity for each material.

second stage with strain hardening. The pressure sensitivity, defined as $d \Sigma / d P$, is 1.31 , which is close to the value we reported for Mg-PSZ. ${ }^{7.11}$

Inasmuch as direct evidence of yielding was found on the tensile surface of a bend specimen, the tensile-yield stress may be obtained by four-point bending. Typically, the tensile-yield stress is only about one-half of the compressive-yield stress. A better way to compare data from different stress states is to express them in terms of effective stress $\left(\sigma_{c}\right)$ and mean stress $\left(\sigma_{m}\right){ }^{3}$ The concept of effective stress and mean stress is well known in stress analysis of plasticity. ${ }^{12}$ Essentially, the effective stress is related to the invariant shear/deviatoric component, whereas the mean stress is the invariant hydrostatic component. In the case of hydraulic compression (including the special case of $P=0$ in uniaxial compression), $\sigma_{e}=\Sigma$, and $\sigma_{m}=-P-\Sigma / 3$. In the case of uniaxial tension, $\sigma_{e}=\Sigma$ and $\sigma_{m}=\Sigma / 3$. Using the above notation, a "yield locus" for Ce-TZP at room temperature is shown in Fig. 9. As apparent from Fig. 9, data for $\sigma_{e}$ versus $\sigma_{m}$ in the negative mean -stress domain follow a linear relationship ${ }^{3}$

$$
\sigma_{e} / \sigma_{c}^{*}+\sigma_{m} / \sigma_{m}^{*}=1
$$

where $\sigma_{c}^{*}$ and $\sigma_{m}^{*}$ are the extrapolated linear intercepts at the two stress axes. Yielding is obtained in transformation plasticity when the stress state falls outside of the $\sigma_{e}^{*}-\sigma_{m}^{*}$ line. From this plot we found the uniaxial tensile yield in four-point bending to be lower than expected from the yield criterion. This could result from a convex curvature of the yield surface in the positive mean-stress domain or, alternatively, from a surface effect which lowers the yield stress in bending. Further studies are needed to clarify this issue.

A shear-dilatant-yield criterion such as the one demonstrated above dictates that both a shear and volumetric strain will be generated upon yielding. ${ }^{3}$ This was confirmed in all of our experiments. For example, in compression, an axial compression, $\varepsilon_{a}$, was accompanied by a radial expansion, $\varepsilon_{r}$, of a comparable or somewhat larger magnitude, creating both an invariant deviatoric and an invariant volumetric-strain component. Following the standard notation, the deviatoric strain can be shown to be $2\left(\varepsilon_{a}+\varepsilon_{r}\right) / 3$, and the volumetric strain to be $2 \varepsilon_{r}-\varepsilon_{a}$. In Table I, we tabulate the strain components from two samples deformed to two different stages. It can be seen that the deviatoric and the volumetric components are roughly equal, which is consistent with a linear yield criterion of $\sigma_{e}^{*} \approx \sigma_{m}^{*}$. The above consis-

Table I. Plastic Strains in Transformation Plasticity of Ce-TZP $\left(M_{b}=-98^{\circ} \mathrm{C}\right)$

\begin{tabular}{ccccc}
\hline & \multicolumn{4}{c}{ Plastic strain $(\%)$} \\
\cline { 2 - 5 } Stage & Axial & Radial & Deviatoric & Volumetric \\
\hline I & $\mathbf{0 . 8 2 5}$ & 1.085 & 1.273 & 1.345 \\
II & 2.740 & 3.550 & 4.193 & 4.360 \\
\hline
\end{tabular}

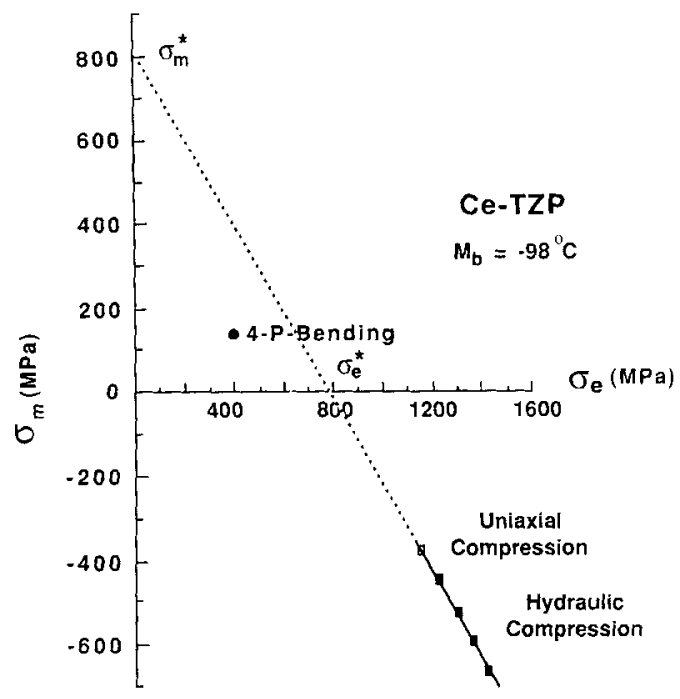

Fig. 9. Effective stress versus mean stress of Ce-TZP under different stress states.

tency between strain components and yield criterion satisfies the so-called normality rule in the theory of plasticity, which is a necessary result of the principle of maximum plastic work. ${ }^{12}$

\section{(4) Temperature Dependence}

The temperature dependence of yield stress in tension, $Y_{f}$, and in compression, $Y_{c}$, shown in Fig. 10 demonstrates a remarkable feature of transformation plasticity in Ce-TZP, namely, a positive temperature dependence and a vanishing yield stress as $M_{h}$ is approached. This positive temperature dependence continues into higher temperatures and persists at all levels of strains.

Whereas the above results may manifest the larger stress assistance which is needed at higher temperatures to provide the requisite driving force for $t$-to-m transformation, deformation at different temperatures may be subject to a different level of thermal activation. If so, the fully compensating effect of stress assistance and chemical driving force could have been obscured somewhat in Fig. 10. ${ }^{13}$ Complications arising from a varying amount of thermal activation can be circumvented by testing transformation plasticity of materials of different $M_{b}$ at the same temperature, since the requisite stress assistance is likely to be determined by the difference between the test temperature $(T)$ and $M_{b}$. For Ce-TZP, $M_{b}$ can be changed by varying the grain size and sintering conditions without modifying the composition. The results on the room-temperature yield stresses for Ce-TZP of different $M_{b}$ are shown in Fig. 11. A constant dependence on $T-M_{b}$ in uniaxial compression, $d Y_{c} / d\left(T-M_{b}\right)=5.54 \mathrm{MPa} /{ }^{\circ} \mathrm{C}$, was obeyed throughout the $M_{b}$ range studied. In the above, $T$ is at room temperature.

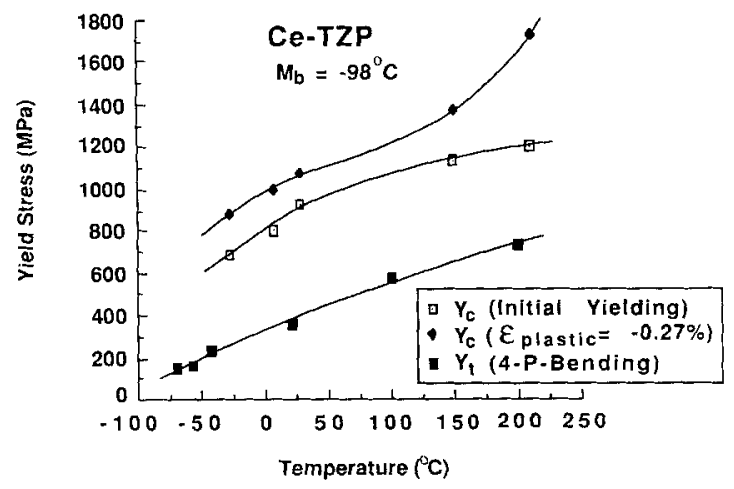

Fig. 10. Positive temperature dependence of yield stress, $Y_{c}$ in compression and $Y_{1}$ in tension, for Ce-TZP. 


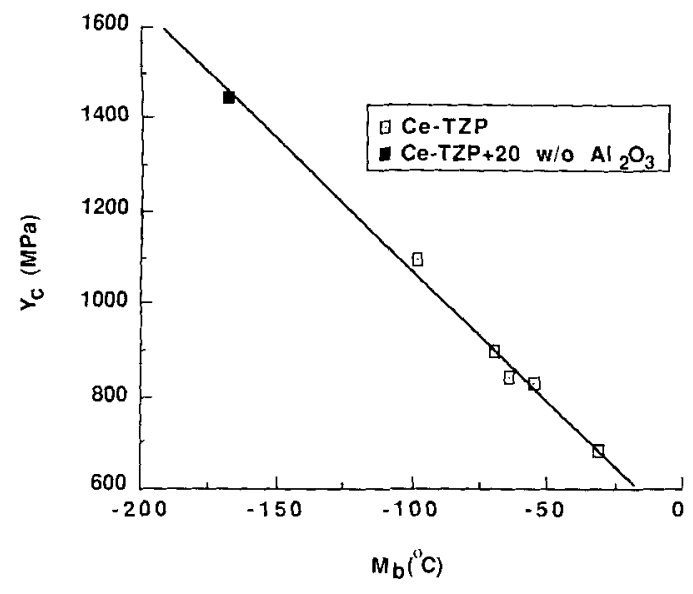

Fig. 11. Compressive yield stress versus $M_{b}$.

Note that in Fig. 11, data of alumina-containing Ce-TZP at $20 \mathrm{wt} \%$ addition were included for comparison. This composite has a Ce-TZP matrix interspersed with alumina inclusions. Compared with Ce-TZP, the burst temperatures were lower, and the bursts were smaller in composites. Transformation plasticity in the composite is entirely attributed to the Ce-TZP matrix and apparently subject to the same stress assistance.

\section{(5) Strain-Rate Sensitivity and Stress Relaxation}

In a further attempt to differentiate the two stages of plastic deformation in transformation plasticity, strain-rate sensitivity and stress relaxation were studied. As shown in Fig. 12, perfect plastic deformation in stage $I$ is rate independent, whereas strainhardening deformation in stage II is highly rate sensitive.

In evaluating the above results, we recognized that stage I deformation was localized and autocatalytic, proceeding at a nearsubsonic velocity. Thus, the strain rate dictated by the loading strain has little to do with the true strain rate in the deformation bands. To circumvent this problem, an alternative method which directly probed the thermally activated nature of deformation in stage I, i.e., stress relaxation, was cmployed. In this technique, the total strain across the specimen is held constant, so that the stress relaxation can be entirely attributed to a forward plastic flow which must be fully compensated by a reverse, elastic springback. Stress relaxation eventually ceases when the remaining applied stress is too low to overcome the internal stress in the deformed body. Since the total amount of plastic-strain increment incurred in stress relaxation is small, the method is thought to probe thermal activation of a constant deformed state under a range of stresses. In Ce-TZP, at room temperatures, we found stress relaxation in stage I to be negligible, whereas that in stage II was very significant. Although further analysis of these results will not be pursued here, it suffices to state that these findings are consistent with

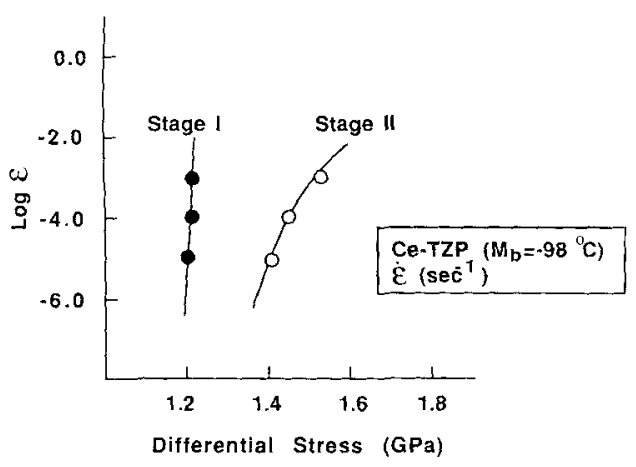

Fig. 12. Yield stress versus strain rate for Ce-TZP during stage I and stage II.
Fig. 12 and verify the distinctly different nature of transformation plasticity in the two stages. ${ }^{7}$

\section{(6) $M_{b}$ and Prior Strain}

The effect of prior strain on $M_{b}$ was found to depend on the stage of deformation as well. In this investigation, samples were deformed in uniaxial compression to various strains, then cooled without a stress to induce further transformation at lower temperatures. As shown in Fig. 13 for a Ce-TZP with $M_{b}=-98^{\circ} \mathrm{C}$, prior deformation in stage I did not affect the burst temperature in subsequent transformation. Prior deformation into stage II, however, eliminated the burst altogether; instead, a gradual transformation occurred below $-98^{\circ} \mathrm{C}$. It should be noted also that during stage $\mathrm{I}$, an interrupted test can be restarted, after unloading, by reloading to essentially the same flow-stress level in stage I as before. These results serve as another illustration of the correspondence between stress assistance and undercooling, namely, a flat flow stress mirrors a constant $M_{b}$ in stage $\mathrm{I}$, whereas the termination of the perfect plastic deformation reflects the disappearance of the burstlike transformation during cooling. As will be discussed below, constant burst temperature independent of prior strain provides direct evidence of the operation of multiplesite nucleation events in autocatalysis.

\section{Stress-Assisted Transformation Plasticity}

To provide a basis for stress assistance, we first turn to a continuum analysis drawing on the results of macroscopic plastic deformation and equilibrium thermodynamics.

From the Clausius-Clapeyron equation, we obtain ${ }^{14}$

$$
d P / d T=\Delta S / \Delta V=\Delta S / V e_{T}^{*}
$$

where $\Delta S$ and $\Delta V$ are the entropy difference and volume difference, respectively, between the $t$ and $m$ phases, $V$ is the volume of the $t$ phase, and $e_{T}^{*}$ is $\Delta V / V$. Using the above relation, and the definition of pressure sensitivity, $\alpha_{c}$, of the yield stress in compression, $Y_{c}, d Y_{c} / d P=\alpha_{c}$, we have

$$
d Y_{c} / d T=\alpha_{c} \cdot \Delta S / V e_{T}^{*}
$$

If we use $\Delta S=4.175 \mathrm{~J} / \mathrm{mol}$ for pure zirconia, $V=2.063 \times$ $10^{-5} \mathrm{~m}^{3} / \mathrm{mol}$ for Ce-TZP, $e_{T}^{*}=0.05$ for highly deformed $\mathrm{Ce}$ -

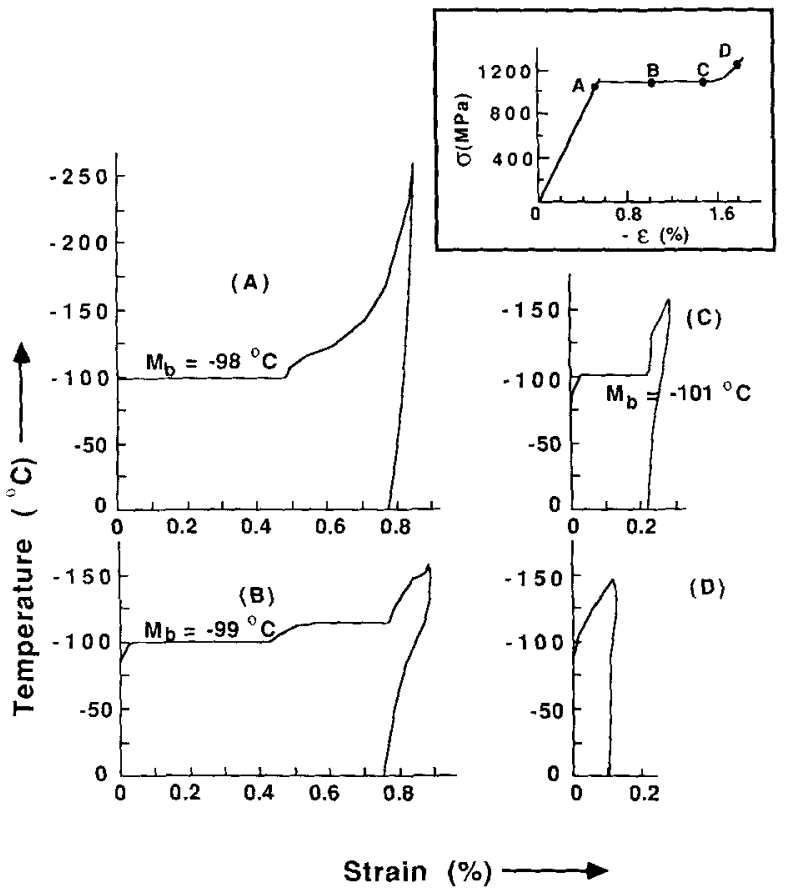

Fig. 13. Effect of prior strain on dilatometry curve. 
TZP, and $\alpha_{r^{\prime}}=1.31$ from Fig. 8 , then the predicted temperature sensitivity is $5.30 \mathrm{MPa} / \mathrm{K}$. This prediction is in reasonable agreement with the observed temperature sensitivity of compressive yield (Figs. 10 and 11).

To compute the temperature sensitivity of tensile yield, one can similarly define a pressure coefficient $\alpha_{r}$, for the yield stress in tension, $Y_{t}$, such that an equation similar to Eq. (3) may apply. Since a direct measurement of $\alpha$, using hydraulic tensile tests was not attempted in the present study, we will estimate its value from the data shown in Fig. 9 using the linear yield criterion given by Eq. (1). In a hydraulic tensile test, $\sigma_{e}=\Sigma, \sigma_{m}=-P+\Sigma / 3$. Substitution into the yield criterion, with $Y_{t}=\Sigma$ at yielding, finds

$$
d Y_{t} / d P=\alpha_{1}=3 \sigma_{e}^{*} /\left(3 \sigma_{m}^{*}+\sigma_{e}^{*}\right)
$$

From Fig. 9, $\sigma_{m}^{*}=770 \mathrm{MPa}$ and $\sigma_{e}^{*}=800 \mathrm{MPa}$, giving $\alpha_{t}=0.772$. The calculated temperature sensitivity from

$$
d Y_{t} / d T=\alpha_{t} \Delta S / V e_{T}^{*}
$$

is $3.12 \mathrm{MPa} / \mathrm{K}$, which is also in reasonable agreement with the data in Fig. 10. Indeed, from Eqs. (3) and (5), we find $Y_{r} / Y_{c}=0.589$, which is approximately obeyed by the data of Fig. 10 throughout the temperature range investigated.

While the above macroscopic picture establishes the correspondence of stress assistance to the chemical driving force and to verify the self-consistency between the stress-state sensitivity and temperature sensitivity, a microscopic picture connecting stress assistance with transformation can offer further insight. This is attempted below. We recognize that the effect of the mechanical work done by a set of external stresses on the transformation process depends on two factors: (i) the transformation strain of the nucleus and (ii) the relative orientation of the nucleus with respect to the stress axes. The coupling of the applied stress and the transformation strain determines the stress assistance to transformation. ${ }^{15}$

Presumably, martensitic transformation in Ce-TZP is nucleation controlled, at least initially. Thus, the above coupling is most critical at the nucleation stage. A detailed analysis of experimental evidence of coherent nucleation in pure $\mathrm{ZrO}_{2}$ by Chiao and Chen ${ }^{16-19}$ has provided the following picture. ${ }^{19}$ The nucleus lies close to the $(100)$, plane, with a dilatation of $2 \%$ across and a shear of $16 \%$ nearly along $[001]_{t}$. This habit plane maintains coherency but is slightly elongated along [010] by $2.3 \%$, so that the invariant plane strain (IPS) condition is violated. In the present analysis, we shall treat the latter in-plane distortion as isotropic in the plane for simplicity. Three strains are thus assigned for a nucleus: the shear strain on the habit plane, $s_{T}$, the normal strain across the habit plane, $e_{T}$, and the total volumetric strain, $e_{T}^{*}$, as schematically shown in Fig. 14(A). Referring to Fig. 14(B), we specify the configuration for mechanical coupling to the nucleus, which is itself defined by its habit normal, $\mathbf{n}$, and the two equivalent shear directions, $\mathbf{s}$ or $-\mathbf{s}$, by two angles. The first is the angle, $\psi$, be tween $\mathbf{n}$ and the stress axis, $\mathbf{z}$; the second is the angle, $\chi$, between $\mathbf{s}$ or $-\mathbf{s}$ and the maximum shear stress along $\mathbf{n} \times(\mathbf{z} \times \mathbf{n})$. For the stress state, in addition to a differential stress, $\Sigma$, a pressure, $P$, is also applied. (In this part of the paper, we will assign a positive sign to a tensile differential stress and a negative sign to a compressive differential stress.)

The mechanical work, $W$, which couples the transformation strain and the applied stress is evaluated in the Appendix. Its value depends on angles $\psi$ and $\chi$, which can assume various values for different nuclei. Two extreme cases are considered here. First, the maximal coupling is of interest, since it would provide the maximal driving force to trigger the transformation. By choosing a set of angles to maximize $W$ (see the Appendix), we find

$$
\begin{aligned}
W_{\max }= & -P e_{T}^{*}+|\Sigma|\left\{s_{T}+(\Sigma /|\Sigma|)\left(e_{T}^{*}+e_{T}\right) / 2\right. \\
& \left.+[(\Sigma /|\Sigma|)-1 / 2]\left(3 e_{T}-e_{T}^{*}\right)^{2} / 4 s_{T}\right\} / 2
\end{aligned}
$$

where the higher-order terms of the order of (excess normal strain $)^{4} /(\text { shear strain })^{3}$ and smaller have been ignored. In this extreme, the anticipated pressure sensitivity for transformation plas-
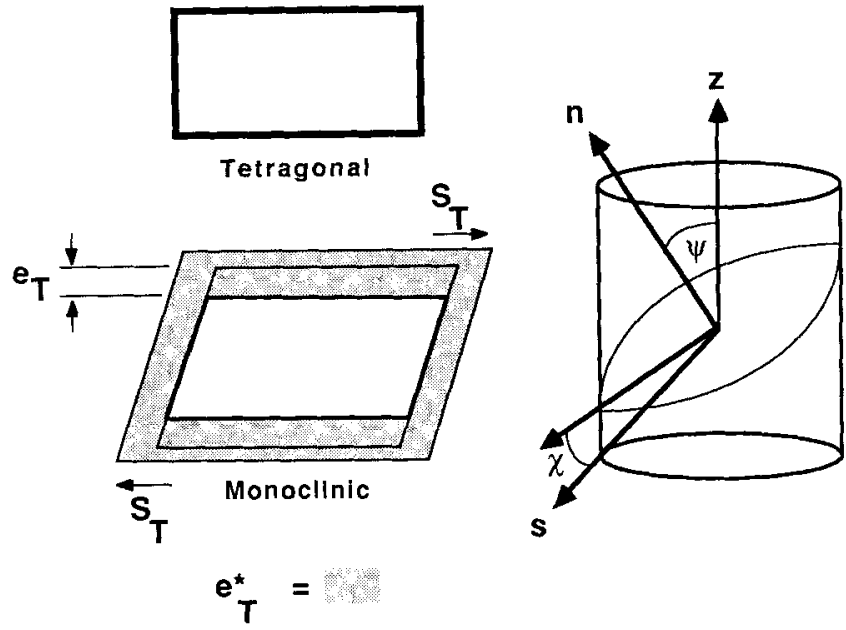

Fig. 14. (A) Three transformation strains assigned for a martensite nucleus. $(B)$ The configuration for mechanical coupling to the nucleus

ticity, calculated from $\alpha=d|\Sigma| / d P$ from Eq. (11) at a constant $W_{\max }$, would be

$$
\alpha=2 e_{T}^{*} /\left[s_{T}+\left(e_{T}^{*}+e_{T}\right) \Sigma / 2|\Sigma|\right]
$$

In the above, a subscript of $t$ or $c$ may be assigned, depending on the sign of $\Sigma$. The above case would be realized if autocatalysis could be triggered by a single nucleus. On the other hand, as will be discussed in the next section, we may envisage each deformation band to be triggered by the operation of a multiple-site nucleation event, which is required to satisfy, for example, the condition of percolation. In such an event, it is highly unlikely, statistically, for all those participating nuclei to have the mostfavorable configuration to receive the maximum stress assistance. Rather, a random sampling of nuclei of all configurations would be more realistic. The stress assistance in the latter case is the average $W$ for all possible $\psi$ and $\chi$. The computed stress assistance (see the Appendix) then becomes

$$
\left.W_{\text {avg }}=-P e_{T}^{*}+|\Sigma|\left[2 s_{T} / \pi\right)+(\Sigma /|\Sigma|) e_{T}^{*}\right] / 3
$$

The corresponding prediction of pressure sensitivity is

$$
\alpha=3 e_{T}^{*} /\left[2 s_{T} / \pi+e_{r}^{* \Sigma} /|\Sigma|\right]
$$

Because of the orientation optimization, the pressure sensitivity deriving from $W_{\max }$ should be smaller than the other case. Using $s_{T}=0.16, e_{T}^{*}=0.05=2 e_{T}$ for Ce-TZP, the calculated $\alpha_{c}$ is 2.89 and 0.816 from $W_{\text {avg }}$ and $W_{\max }$. As might be expected, these estimates of extreme cases bracket the observed pressure sensitivity of 1.31 ; i.e., an intermediate case is in operation. This suggests that the group of nuclei responsible for triggering a deformation band is, statistically, only partially biased toward the most-favorable orientation. The available stress assistance in transformation plasticity is between $W_{a x g}$ and $W_{\max }$.

Returning to the earlier continuum picture, we may write, empirically, the stress assistance in hydraulic tension or compression as

$$
W=-P e_{T}^{*}+|\Sigma| e_{T}^{*} /(d|\Sigma| / d P)
$$

In Eq. (10), the last bracket on the right-hand side is simply the pressure sensitivity in hydraulic tension or compression. Since the pressure sensitivity is bracketed by the theoretical estimates of Eqs. (7) and (9), $W$ lies between $W_{\text {avg }}$ and $W_{\text {max }}$. Generalizing this representation to other stress states, it can be shown that the stress assistance is

$$
W=\sigma_{m} e_{T}^{*}+\sigma_{c} e_{T}^{*} /\left(\sigma_{e}^{*} / \sigma_{m}^{*}\right)
$$

The last term in parentheses on the right-hand side in Eq. (11) is 
the pressure sensitivity of the effective yield stress. Knowing the above relation, the temperature shift due to a stress assistance can be computed from $W / \Delta S$.

Before concluding this discussion, we note that the microscopic picture presented above deals with nucleation only. In theory, the stress coupling inherited from the nucleation stage may not be continued during the propagation of a deformation band. Hence, while the yield stress is governed by nucleation considerations, the partitioning of the final macroscopic strains, which depends on the propagation stage as well, need not be consistent with the yield criterion. Our data of strain partitioning into deviatoric and volumetric components suggest, however, that the product of $\left(\sigma_{e^{*}}^{*} / \sigma_{m}^{*}\right)$ and (deviatoric strain/volumetric strain) is close to unity, as shown in Fig. 9 and Table I, respectively. This is the so-called normality condition in plasticity. ${ }^{12}$ Thus, there seems to be a consistent stress assistance operating at all phases of transformation. Further insight into the nature of the stress bias in achieving the strain partitioning was provided by texture analysis, in which we found a clear correlation betwcen stress assistance and the crystallographic orientations of the transforming tetragonal variants. ${ }^{9}$ Although the subject may still need further study, the coupling between the applied stress and the parent variant emerges as a consistent microscopic picture for stress-assisted transformation.

In the above consideration, the notion of autocatalysis triggered by certain critical, multiple-site nucleation events was invoked. As we pointed out in the Introduction, autocatalytic, burstlike transformation is a special feature of homogeneous metastable TZP. This is examined next.

\section{Autocatalysis}

Several observations concerning autocatalysis in stress-assisted transformation and in thermally induced transformation are deemed to be central. First, a homogeneous, metastable matrix and a constant stress or undercooling are required to trigger the burst. Second, even with prior partial autocatalytic transformation, autocatalysis can be reinitiated at essentially the same stress or undercooling as before up to a certain strain. Third, autocatalysis is terminated when the transformable matrix is partitioned into isolated pockets. We believe these observations can be explained, assuming that autocatalysis is initiated by a multiple-nucleation event which, once realized, can stimulate further transformation to propagate rapidly over an extended region. To develop this reasoning, we will focus on the statistics of multiple-nucleation events. Whereas further micromechanical considerations which describe the enhanced stress assistance in the vicinity of transformed variants presumably can define the requisite multiple-nucleation event more specifically, they are not pursued here.

To begin with, a review of the statistics of single-nucleation events is instructive. Current understanding of the $t$-to- $m$ transformation supports a picture of nucleation control ${ }^{16-19}$ in which the probability of nucleation depends on the availability of a nucleating defect. Defects of higher potency can operate at a lower driving force. Thus, the probability of nucleation depends on the undercooling, and the relation of nucleation statistics versus temperature is governed by the potency distribution of nucleating defects. Chen et al. have analyzed the small-particle data and found that the probability of nucleation in isolated particles obeys the extremevalue statistics on the driving force and that the probability increases with the particle size. ${ }^{20}$ The extreme-value exponent of the driving force is small, of the order of 2 . Hence, the temperature dependence of the transformation probability in small particles is rather broad. ${ }^{20}$ When a stress assistance augments the driving force, producing a downward shift of the effective temperature, the nucleation probability is expected to increase with stress in a similar manner. In either case, no abrupt transformation burst at a constant temperature nor perfect plastic deformation at a constant stress is envisaged. Indeed, the gradual transformation below $M_{b}$ and in stage II are characteristic of this type of nucleation statistic of isolated particles, as might be expected from the microstructu at that stage. As transformation proceeds, the most-potent nuclea ing defects available in the isolated pockets operate first, while th remaining, untransformed pockets containing less-potent defec: stay metastable until progressively lower temperatures. The sam exhaustion process of nucleating defects should give rise to strai hardening as observed in stress-assisted transformation.

In contrast, multiple-site nucleation, the postulated mechanisn which triggers autocatalytic transformation in Ce-TZP, can bc shown to have a much narrower stress or temperature distributior for transformation. Consider a transformation band which, once formed, engulfs $N$ grains. The critical event leading to the formation of a transformation band is envisaged to be the nucleation of $n$ variants in $n$ contiguous grains. It may be postulated, following the common assumption in percolation theory, that the attainment of such an event can lead to a nearly spontaneous formation of more transformation variants in all the neighboring grains, and reaching out to engulf all $N$ grains. Let $F$ denote the probability of martensitic nucleation in a single particle/grain. As shown before, ${ }^{20}$ this probability strongly depends on stress and temperature. Since the critical, multiple-site nucleation event involves $n$ nucleation sites, its probability, $F^{*}$, should be proportional to $F^{n}$. Since such a critical event can come from any $n$ contiguous grains within the group of $N$ grains, a very large number of distinguishable configurations needs to be enumerated. Taking the above two factors into account, we have evaluated the probability of a multiple-site nucleation event and found it to be approximately

$$
F^{*}=N z^{n} F(\Sigma, T)^{n} / n
$$

in which $z$ is of the order of the grain-coordination number in a polycrystal.

Equation (12), pertaining to multiple-site nucleation statistics, readily explains our experimental observations concerning autocatalysis. First, it predicts that the probability of autocatalytic transformation can be extremely sensitive to the driving force, in terms of temperature or stress, if the requisite number of nuclei, i.e., $n$, in a critical, multiple-nucleation event is large. This is because the spread of transformation temperature or stress due to $F^{*}$ is essentially $1 / n$ times that of the single-site nucleation due to $F$-a direct result of $F^{*}$ being proportional to $F^{n}$. This prediction explains why the transformation burst is abrupt and occurs at a reproducible temperature or stress value for a given Ce-TZP. Second, it predicts that, for a given $n$, the probability of autocatalytic transformation increases rapidly with $N$-a direct result of $F^{*}$ being proportional to $N$. The magnitude of $N$ is determined by the number of grains in a homogeneous, transformable region. This prediction explains why autocatalysis occurs only in undeformed or less-deformed TZP and why it terminates after a certain fraction of the untransformed materials or prior strain is consumed. In essence, a "size effect" is operating, as evidenced in later stages of transformation when the remaining tetragonal grains in isolated pockets are too few to allow autocatalysis to nucleate statistically, leaving single-site nucleation events to operate as the moredominant process. Since it is reasonable to envisage activation volume to scale with $n$, multiple-site nucleation events in stage II should have a higher activation volume than that of single-site nucleation events in stage $I$. In this connection, we can also rationalize the distinct difference in strain-rate sensitivity and in thermal activation between stages I and II. (The strain-rate sensitivity increases with decreasing activation volume. ${ }^{7,13}$ )

Because of the $N$ dependence, microstructure is expected to play an important role in the operation of autocatalysis. In addition to the microstructural evolution brought about by transformation itself, a second phase can partition the metastable matrix to suppress autocatalysis as well. This was confirmed in Ce-TZP by the addition of alumina. We found, for example, that the addition of $20 \mathrm{wt} \%$ alumina lowered $M_{b}$ by $\approx 70^{\circ} \mathrm{C}$ and reduced the magnitude of the transformation burst. (In the limiting case ot ZTA or MgPSZ, in which the metastable grains or precipitates are individually isolated, no burst was detected at any temperature.) On the other 
hand, grain refinement reduces the size scale but does not alter the phase homogeneity. Consequently, it lowers $M_{b}$ by lowering the single-site nucleation probability ${ }^{20}$ but otherwise does not affect the burstlike characteristics itself (see Fig. 5 of the companion paper $^{5}$ ).

So far in our analysis for autocatalysis, a stress assistance has been regarded as equivalent to undercooling. However, since the stress coupling with transformation shape strains in different grains is not identical, being a function of grain and variant orientations, the application of a stress assistance has a somewhat different statistical effect than undercooling. Specifically, the spatially nonuniform driving force in stress-assisted transformation makes multiple-site nucleation events more difficult to realize statistically. This is because, despite a large applied stress, only very few grains with the most-favorable orientation receive the maximum stress assistance, whereas the rest of the much-less-favorable orientations have much less stress assistance and, hence, a smaller driving force. A wide disparity of nucleation potency among different sites thus exists, which does not lend itself favorably to multiple-site nucleation statistics. In essence, the largely random orientation factor entering the stress assistance introduces an additional random factor to the nucleation statistics. This subtle difference between stress-assisted transformation and thermally induced transformation can be illustrated by the following two observations. We found that, in $\mathrm{Ce}-\mathrm{TZP}$ with $20 \mathrm{wt} \% \mathrm{Al}_{2} \mathrm{O}_{3}$, the stress-strain curve had no perfect plastic regime, yet the $M_{b}$ and the transformation burst were quite distinct in cooling. We also found that autocatalysis becomes increasingly difficult at higher temperatures, despite a larger stress assistance at yielding which essentially compensates for the loss of chemical driving force. (See Eq. (3).) These observations suggest that, with a lower, background chemical driving force or in a somewhat partitioned microstructure, the mere provision of a higher stress assistance alone may not be adequate to trigger autocatalysis. Finally, the orientationally random factor also broadens the statistics of single-site nucleation in stress-assisted transformation. Thus, especially at higher temperatures, the driving force in the optimally biased grains may be large enough to favor single-site nucleation, which could be further facilitated by thermal activation. In this way, the sharp, two-stage stress-strain curve at lower temperature and homogeneous TZP gradually evolves into a smooth stress-strain curve at higher temperatures and in composites.

\section{Implications on Transformation Plasticity and Transformation Toughening}

The major findings of this study, the dominance of stress assistance and autocatalysis in $\mathrm{Ce}-\mathrm{TZP}$, are expected to be general for homogeneous $t$-zirconia polycrystals near their $M_{b}$ temperatures. For such ceramics, we expect to find a positive pressure sensitivity, a large tension--compression strength differential, a positive temperature sensitivity, and formation of shear bands in transformation plasticity. Although not within the bounds of this study, we have also contrasted the above behavior to the other extreme, which is most easily realized in a two-phase microstructure or at higher temperatures. 7,11 The second phase can be due to either a nonzirconia phase or a nontransforming phase. Even though a positive pressure sensitivity and a large strength differential in tension and compression may still be characteristic of such ceramics, they have a much-less-positive or even a negative temperature sensitivity in transformation plasticity. ${ }^{7,11}$ They show no macroscopic shear bands either. Depending on the magnitude of the chemical driving force and the prior deformation history, a TZP may behave in either way. ${ }^{7,11}$ Superficially, the latter extreme appears to correspond to the case of strain-induced transformation, ${ }^{21.22}$ in which nucleation sites are classically provided by deformation via dislocations and slip-band intersections. ${ }^{18,23}$ This picture is suspect in view of the ubiquitous evidence of stress-state sensitivity among zirconia ceramics, as we pointed out elsewhere. ${ }^{7,11}$ Further research is needed to more carefully clarify this issue.
The formation of deformation bands merits special discussion in relation to its implication on strength and fracture of this class of ceramics. Deformation bands are inevitable for perfect plastic deformation, which offers no resistance to flow localization. Another consequence of the perfect plastic deformation is that the location and development of deformation bands are very sensitive to the boundary condition. Because many bands begin to form upon the initial load drop, general yielding usually precedes the formation of the main crack. For example, the deformation bands shown in Fig. 6 do not contain the main crack, which is to the right of these bands but not shown in the figure. Indeed, when several indents were introduced to a bend specimen which is then loaded to fracture, all but one had no visible cracks, even though deformation bands emanated from corners of all the indents. Thus, crack nucleation is definitely preceded by the full development of flow localization.

Caution must therefore be exercised in relating the process zone width to the fracture toughness because the observed process zone near the fracture surface is not only due to crack propagation. Since a crack is nucleated after full development of a deformation band, most of the plastic strain in the band does not contribute to the fracture energy. This is illustrated in Fig. 15 for two bend specimens: The specimen shown on the left has an indent from which the bending fracture initiated; the specimen on the right has no indent but was simply bent to fracture. Note that the shear band on the right is away from the fracture surface, yet it has a width comparable to that of the band on the left which falls directly on the fracture path. We also note that, even with a controlled flaw, the flaw-initiated flow localization in a perfect plastic solid is still sensitive to the boundary condition. Thus, flaw-initiated deformation is no longer solely determined by flaw geometry and nominal stresses, and, in this sense, the notion of small-scale yielding in fracture mechanics has been violated. Such is the case for Ce-TZP.

To verify the above expectation, we measured the fracture toughness at room temperature and found it to be $10 \mathrm{MPa} \cdot \mathrm{m}^{1 / 2}$. We have also derived a modified McMeeking-Evans equation relating toughness increment and the transformation-zone height, ${ }^{24}$ using the shear-dilatant yield criterion (Eq. (1)) in the evaluation of plastic work due to transformation plasticity. The predicted toughness increment $\Delta K$ is s $^{7,11}$

$$
\Delta K=0.48 E f e_{T}^{*} h^{1 / 2} /(1-\nu)
$$

for $\sigma_{m}^{*} \approx \sigma_{e}^{*}$. In the above, $E$ is Young's modulus, $\nu$ is Poisson's ratio, $f$ is the fraction of transformation, and $h$ is the height of the transformation zone. Assuming $E$ is $190 \mathrm{GPa}, \nu$ is $0.325, e_{T}^{*}$ is $0.05, f$ is 0.3 , and $h$ is 0.1 to $1 \mathrm{~mm}$ as observed, the estimated toughness increment is from 20.3 to $64.2 \mathrm{MPa} \cdot \mathrm{m}^{1 / 2}$. Comparing it with the measured toughness, we conclude that only a small fraction, estimated to be between $1 / 4$ and $1 / 40$, of the total plastic work dissipated in the observed transformation zone was actually incurred during crack propagation.

The reason for crack nucleation in deformation bands is not difficult to understand. Deformation bands in Ce-TZP must carry a large shear component, as expected from the $t$-to- $m$ transformation and the macroscopic shear strains. Such concentrated shear deformation can generate microcracks along grain boundaries. Grain-boundary microcracks have been reported for deformed Mg-PSZ ${ }^{3,7}$ By acoustic measurement of the elastic-stiffness tensor, we have also obtained direct evidence of a large population of microcracks in Ce-TZP. ${ }^{7}$ Later, during strain hardening, the microcrack-weakened structure fails by the propagation and linkage of microcracks. In this sequence, transformation plasticity in Ce-TZP is accumulating damage from the very start of transformation.

Chen and Reyes-Morel have previously evaluated the criterion for transformation plasticity in zirconia-containing ceramics. ${ }^{3}$ They suggested that the strength of high-toughness zirconia is flaw tolerant but yield limited by the profuse formation of microcracks when transformation yield stress is exceeded. Although their initial example was an $\mathrm{Mg}$-PSZ, the Ce-TZP studied here behaves in a 

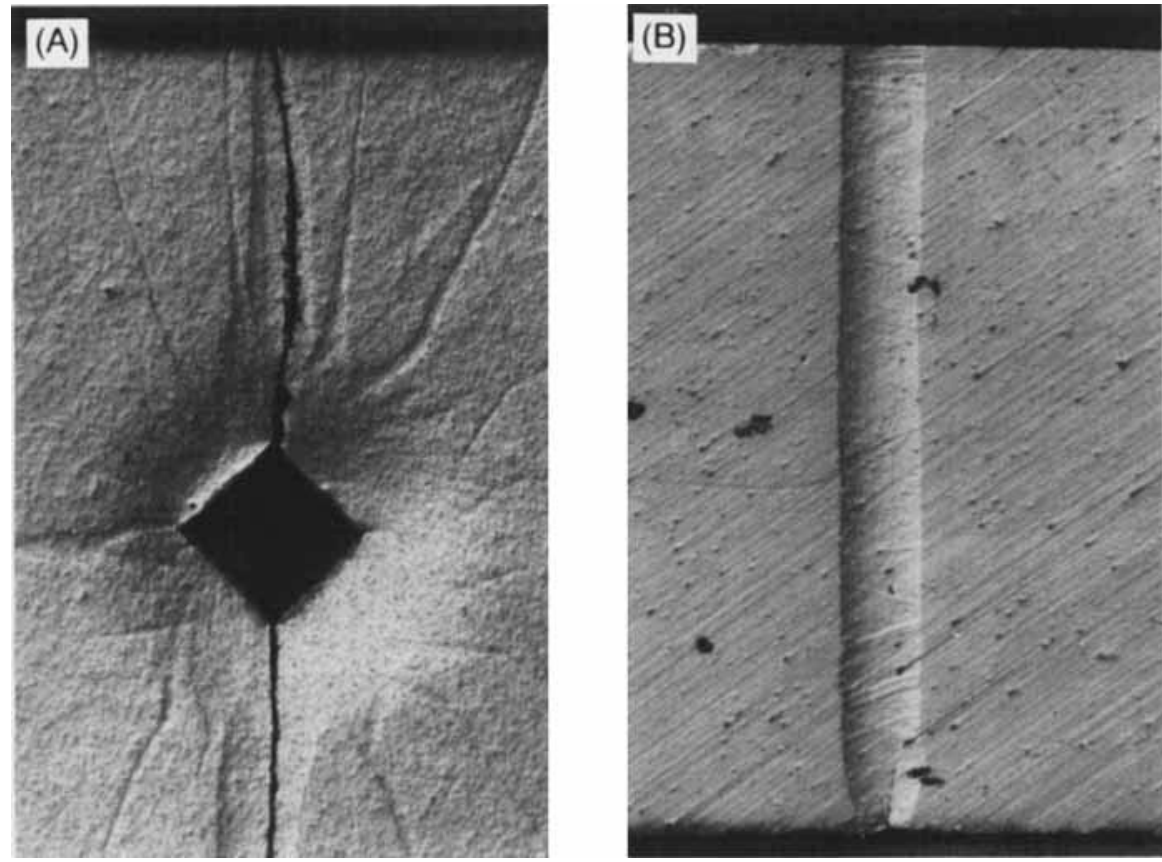

\section{$1 \mathrm{~mm}$}

Fig. 15. Deformation bands on tensile surfaces: $(A)$ bend specimen with an indent; $(B)$ bend specimen without an indent (away from the fracture surface).

similar way. Inasmuch as the strength is limited by yielding, and yielding in transformation plasticity is highly sensitive to temperature and stress state, engineers contemplating using transformation-toughened zirconia ceramics for strength-limited applications must pay extreme attention to this design aspect.

\section{Conclusions}

Transformation plasticity in Ce-TZP is stress assisted. The stress-strain curve at lower temperatures has a one-to-one correspondence to the transformation curve obtained by cooling through $M_{b}$. The flow stress has a positive temperature dependence, sensitive to the stress state, as expected for the stress assistance to a shear-dilatant martensitic transformation.

When the burstlike transformation occurs in transformation plasticity, perfect plastic deformation is attained. Such deformation is mechanically unstable, as manifested by the presence of a load drop and many deformation bands. This mode of deformation is eventually terminated after a substantial strain has been accumulated, followed by a strain-hardening regime. The strainrate dependence is weak in the perfect plastic regime but strong in the strain-hardening regime.

While strain hardening and a stronger strain-rate sensitivity in stage $\mathrm{H}$ are definite characteristics of single-site nucleation events, the autocatalytic, burstlike transformations during stage I are different and triggered by multiple-site nucleation events. Considerations of multiple-site nucleation statistics successfully explain the sharp burst temperature and the constant flow stress, as well as the absence of autocatalysis in later stages of transformation and in composites. It is also found that autocatalysis cannot be realized in transformation plasticity at higher temperatures.

The severe damage accumulated during localized deformation prevents tensile deformation of Ce-TZP much beyond initial yielding. Fracture originates from one of the fully developed deformation bands. In general, the width of the deformation band is not determined by the crack-tip-stress field. Thus, only a small fraction of the total plastic work in the transformation zone is actually attributable to crack propagation.

\section{APPENDIX}

Referring to Fig. $14(A)$, the transformation strain includes a volume strain, $e_{T}^{*} ;$ an in-plane dilatation, $\left(e_{T}^{*}-e_{T}\right) / 2 ;$ a normal strain, $e_{T}$; and a shear strain, $s_{T}$. To match the in-plane dilatation, we further decompose the normal strain into $\left(e_{T}^{*}-e_{T}\right) / 2$ and $\left(3 e_{T}-e_{T}^{*}\right) / 2$. With respect to these strains, the mechanical work, $W$, is now computed from the three components below.

(1) A pressure work, which couples $P$ and the volume dilatation, $e_{T}^{*}$, is given by

$$
W_{1}=-P e_{T}^{*}
$$

(2) A pressure work, which couples $\Sigma$ and the dilatation, $\left(e_{T}^{*}-e_{T}\right) / 2$, is given by

$$
W_{2}=\Sigma\left(e_{T}^{*}-e_{T}\right) / 2
$$

(3) The excess normal strain, $\left(3 e_{T}-e_{T}^{*}\right) / 2$, and the shear strain, $s_{T}$, on the habit plane are of IPS type. Their coupling with $\Sigma$ can be evaluated by employing a Mohr circle construction, as previously performed by Patel and Cohen. ${ }^{15}$ This work is given by

$$
\left.W_{3}=|\Sigma| s_{T} \sin \psi \cos \psi \cos \chi+\Sigma\left[3 e_{T}-e_{T}^{*}\right) / 2\right] \cos ^{2} \psi
$$

The total work, $W$, is given by

$$
W=W_{1}+W_{2}+W_{3}
$$

Note that only $W_{3}$ above is orientation dependent.

The most favorable angles are found by setting $\chi=0$ and optimizing $W_{3}$ with respect to $\psi$. This gives

$$
\psi=\frac{1}{2} \tan ^{-1}\left\{(\Sigma /|\Sigma|)\left[2 s_{T} /\left(3 e_{T}-e_{T}^{*}\right)\right]\right\}
$$

Thus, the maximum stress assistance is obtained by substituting Eq. $(\mathrm{A}-5)$ and $\chi=0$ into Eq. $(\mathrm{A}-3)$ and Eq. $(\mathrm{A}-4)$. The result is given in Eq. (6). We also computed the average $W$ by allowing the nucleus to assume all possible orientations. Thus, both $\psi$ and $\chi$ span from 0 to $\pi / 2$. Taking into account the proper solid angle, the computed average $W$ is given in Eq. (8). 
Acknowledgments: We are grateful for the assistance of Mr. J-S. Cherng in the experimental work and in the preparation of the manuscript, and for useful discussions with Dr. K. J. Bowman.

\section{References}

'R. C. Garvie, R. H. Hannink, and R. T. Pascoe, "Ceramic Steel'," Nature (London), 258 [5537] 703-04 (1975)

${ }^{2} \mathrm{~K}$. Tsukuma and M. Shimada, "Strength, Fracture Toughness, and Vickers Hardness of $\mathrm{CeO}_{2}$-Stabilized Tetragonal $\mathrm{ZrO}_{2}$ Polycrystals (Ce-TZP),"J. Mater. Sci., 204 [4] $1178-84(1985)$

${ }^{3}$ I-W. Chen and P. E. Reyes-Morel, "Implications of Transformation Plasticity in $\mathrm{ZrO}_{2}$-Containing Ceramics: I. Shear and Dilatation Effects," J. Am. Ceram. Soc., 69 [3] 181-89 (1986).

${ }^{4}$ Advances in Ceramics, Vol. 3, Science and Technology of Zirconia. Edited by A. H. Heuer and L. W. Hobbs. American Ceramic Society, Columbus, OH, 1981 ${ }^{5}$ P. E. Reyes-Morel, J.S. Cherng, and I-W. Chen, "Transformation Plasticity of Ce-TZP: II, Pseudoelasticity and the Shape Memory Effect"; to be published in J. Am. Ceram. Soc

${ }^{6} \mathrm{M}$. S. Paterson, Experimental Rock Deformation-The Brittle Field, SpringerVerlag, Berlin, 1978.

'P. E. Reyes-Morel, "An Experimental Study of Constitutive Relations of Transformation Plasticity in Zirconia-Based Ceramics"; Ph.D. Thesis. Department of Nuclear Engineering, Massachusetts Institute of Technology, Cambridge, MA, June 1986.

${ }^{8}$ J. D. Eshelby, "Determination of the Elastic Field of an Ellipsoidal Inclusion and the Related Problems," Proc. R. Soc., London, A, 241, 376-96 (1957).

${ }^{9}$ P. E. Reyes-Morel, P. Yang, B.-S. Li, T. Y. Tien, and I-W. Chen, "Domain Switching as a Toughening Mechanism"; for abstract see p. 94 in 89 th Annual Meeting Abstracts. The American Ceramic Society, Westerville, OH, 1987.

J. W. Rudnicki and J. R. Rice, "Condition for the Localization of Deformation in

Pressure-Sensitive Dilatant Materials,"J. Mech. Phys. Solids, 23 [6] 371-94 (1975).

${ }^{11}$ I-W. Chen and P. E. Reyes-Morel, "Transformation Plasticity and Transformation Toughening in Mg-PSZ and Ce-TZP"; pp. 75-88 in Advanced Structural Ceramics.
Edited by P. F. Becher, M. V. Swain, and S. Sōmiya. Materials Research Society Symposium Series, Materials Research Society, Pittsburgh, PA, 1986.

${ }^{12} \mathrm{R}$. Hill. The Mathematical Theory of Plasticity. Clarendon Press, Oxford, 1950

${ }^{13} \mathrm{I}$-W Chen "Implication of Transfornation Plasticity on Deformation and Fracture Control of Zirconia-Containing Ceramics"; pp. 49-63 in Zirconia Ceramics, Vol. 7. Edited by S. Sōmiya and M. Yoshimura. Uchida Rokakuho Publishing Co., Tokyo, 1986.

${ }^{14}$ E. D. Whitney, "Effect of Pressure on Monoclinic-Tetragonal Transition of Zirconia: Thermodynamics," $J$. Am. Ceram. Soc., 45 [12] 612-13 (1962).

$15 \mathrm{~J}$. R. Patel and M. Cohen, "Criterion for the Action of Applied Stress in the Martensitic Transformation," Acta Metall., 1 [9] 531-38 (1953).

${ }^{16} \mathrm{~J}-\mathrm{W}$. Chen and Y-H. Chiao, "Martensitic Nucleation in $\mathrm{ZrO}_{2}, "$ Acta Metall. 31 [10] 1627-38 (1983).

${ }^{17} \mathrm{I}-\mathrm{W}$. Chen and $\mathrm{Y}-\mathrm{H}$. Chiao, "Martensitic Transformation in $\mathrm{ZrO}_{2}$ and $\mathrm{HfO}_{2}-$ An Assessment of Small-Particle Experiments with Metal and Ceramic Matrices"; pp. 33-45 in Advances in Ceramics, Vol. 12, Science and Technology of Zirconia II. Edited by N. Claussen, M. Rühle, and A. H. Heuer. American Ceramic Society. Columbus, $\mathrm{OH}, 1984$.

${ }^{18} \mathrm{I}-\mathrm{W}$. Chen and Y-H. Chiao, "Theory and Experiment of Martensitic Nucleation in $\mathrm{ZrO}_{2}$-Containing Ceramics and Ferrous Alloys," Acta Metall., 33 [10] 1827-45 (1985).

${ }^{19}$ Y-H. Chiao, "Nucleation and Growth Processes of Martensitic Transformation in $\mathrm{ZrO}_{2}$ Particles"; Ph.D. Thesis. Department of Materials Science and Engineering, Massachusetts Institute of Technology, Cambridge, MA. Sept. 1986.

${ }^{20} \mathrm{I}-\mathrm{W}$. Chen, Y-H. Chiao, and K. Tsuzaki, "Statistics of Martensitic Nucleation." Acta Metall. 33 [10] 1847-59 (1985).

${ }^{21}$ G. B. Olson and M. Cohen, "A Mechanism for the Strain-Induced Nucleation of Martensitic Transformation," J. Less-Common Merals, 28, 107 (1973).

${ }^{22} \mathrm{G}$. B. Olson, "Transformation Plasticity and the Stability of Plastic Flow"; pp. 391-424 in Deformation, Processing, and Structures. Edited by G. Krauss. American Society for Metals, Metals Park, OH, 1982

${ }^{23}$ T. Suzuki, H. Kojima, K. Suzuki, K. Hashimoto, and M. Ichihara, "An Experimental Study of the Martensite Nucleation and Growth in 18/8 Stainless Steel," Acta Metall, 25 [10] 1151-62 (1977).

${ }^{24}$ R. M. McMeeking and A. G. Evans, "Mechanisms of Transformation Toughening in Brittle Materials," J. Am. Ceram. Soc., 65 [5] 242-46 (1982). 